\title{
EQUALITY CASES OF INEQUALITIES INVOLVING GENERALIZED CSISZÁR AND TSALLIS TYPE $f$-DIVERGENCES
}

\author{
MAREK NIEZGODA
}

Abstract. In this note, we study the problem of equality case of two inequalities involving generalized Csiszár $f$-divergences and generalized Tsallis $f$-divergences, respectively, with a convex function $f$. To this end we use generalized inverses of matrices and inverse-positive matrices.

Mathematics subject classification (2010): 94A17, 26D15, 15B48.

Keywords and phrases: Convex function, Csiszár $f$-divergence, Tsallis type $f$-divergence, positive (entrywise) matrix, generalized inverse of matrix, inverse-positive (entrywise) matrix.

\section{REFERENCES}

[1] I. CSISZÁR, Information-type measures of differences of probability distributions and indirect observations, Studia Sci. Math. Hung. 2 (1967), 299-318.

[2] I. CSISZÁR AND J. KÖRner, Information Theory: Coding Theorems for Discrete Memory-less Systems, Academic Press, New York, 1981.

[3] S. S. DRAGOMIR, Upper and lower bounds for Csiszár $f$-divergence in terms of the Kullback-Leibler distance and applications, Inequalities for the Csiszár $f$-divergence in Information Theory, (Ed. S. S. Dragomir), 2000, http://rgmia.vu.edu.au/monographs/csiszar.htm.

[4] S. Furuichi AND F.-C. Mitroi, Mathematical inequalities for some divergences, Physica A, 391 (2012), 388-400.

[5] T. FURUTA, Parametric extensions of Shannon inequality and its reverse one in Hilbert space operators, Linear Algebra Appl. 381 (2004), 219-235.

[6] H. Is a, M. Ito, E. Kamei, H. Tohyama And M. Watanabe, Generalizations of operator Shannon inequality based on Tsallis and Rényi relative entropies, Linear Algebra Appl. 439 (10) (2013), $3148-3155$.

[7] P. A. Kluza And M. NieZgoda, Inequalities for relative operator entropies, Electron. J. Linear Algebra 27 (2014), 851-864.

[8] P. KluZa And M. NiezGoda, Generalizations of Crooks and Lin's results on Jeffreys-Csiszár and Jensen-Csiszár f-divergences, Physica A, 463 (2016), 383-393.

[9] P. KluZA AND M. NIEZGoda, On Csiszár and Tsallis type $f$-divergences induced by superquadratic and convex functions, Math. Inequal. Appl. 21 (2018), 455-467.

[10] M. NiEZGODA, On Shannon like inequalities for $f$-connections of positive linear maps and positive operators, Linear Algebra Appl. 481 (2015), 186-201.

[11] M. NiezGodA, Vector joint majorization and generalization of Csiszár-Körner's inequality for $f$ divergence, Discrete Applied Math. 198 (2016), 195-205.

[12] J. E. PERIS, A new characterization of inverse-positive matrices, Linear Algebra Appl. 154-156 (1991), 45-58.

[13] C. R. RaO AND S. K. Mitra, Generalized Inverse of Matrices and its Applications, John Wiley and Sons, New York, 1971.

[14] K. Yanagi, K. KuriYama And S. Furuichi, Generalized Shannon inequalities based on Tsallis relative operator entropy, Linear Algebra Appl. 394 (2005), 109-118. 